\title{
DENSITY IRREGULARITY OF THE INNER CORONA DETERMINED FROM SIMULTANEOUS MEASUREMENTS OF THE XUV AND THE K CORONAL BRIGHTNESS
}

\author{
M. GUHATHAKURTA \\ G.J. ROTTMANN \\ Laboratory for Atmospheric \\ and Space Physics \\ U. of Colorado, Campus Box 392 \\ Boulder, CO, 80309-0392 \\ R.R. FISHER \\ High Altitude Observatory \\ National Center for Atmospheric Research \\ PO Box 3000 \\ Boulder, CO 80307-3000
}

\author{
F.Q. ORRALL \\ Institute for Astronomy \\ University of Hawaii \\ 2680 Woodlawn Drive \\ Honolulu, HI 96822
}

\begin{abstract}
In this paper we report preliminary results from a study of the inner corona based on the direct comparison of XUV resonance emission line $\lambda 174.53 \mathrm{FeX}$ with that of the white-light emission from the K corona. The data sets were obtained $17 / 18^{\text {th }}$ of March, 1988, during a total solar eclipse of the sun and consists of co-spatial and co-temporal measurements of these two quantities as a function of position angle and height above the solar limb. The local emission of a coronal resonance line is proportional to the electron density squared, the chemical abundance, and the electron kinetic temperature, while the white-light emission (arising from Thomson scattering by electrons) depends directly on the electron density and the local radiation field. Taken together these measurements yield an estimate of the quantity $\left(\mathrm{n}^{2}\right) /(\mathrm{n})^{2}$ where $\mathrm{n}$ is the electron density. This quantity, called "X the coronal irregularity factor" by C.W. Allen, is found to be $>1$.
\end{abstract}

Several studies of the density irregularity factor in the low solar corona have been conducted and the subject remains one of active inquiry. The most recent study of the irregularity has been carried out by Orrall et al. (1990) who used co-spatial and co-temporal observations of the intensity of $\lambda 625 \mathrm{MgX}$ and $\mathrm{pB}$ (polarized brightness) of the $\mathrm{K}$ corona as a function of height above the limb to derive $\mathbf{X}$ for an active and a quiet region on the sun. In the present study we use co-spatial and co-temporal observations of the intensity I of $\lambda 174.53 \mathrm{FeX}$ line and K-coronal brightness B as a function of height above the limb for two positions in the north polar region where the corona appeared relatively free of structures. A description of the XUV imager instrument and its photometric calibration has been given by Guhathakurta, M. (1989) and Rottman, G.J. (1989). The white-light coronal camera with a CCD detector and its calibration has been described by Fisher, R.R. (1989).

A model corona that is spherically symmetric, isothermal, and in hydrostatic equilibrium is used to estimate the XUV emission line intensity and the white-light emission brightness of the corona as a function of height above the limb. We use the same notation and approximations as Guhathakurta, M. (1989), and Orrall et al.(1990). Since XUV intensity, I, varies as $\left(\mathrm{n}^{2}\right)$ and white-light brightness, B, varies as $(n)^{2}$ we can write the equation for $\mathrm{X}$ as follows:

$$
X \equiv \frac{\overline{\left(n^{2}\right)}}{(\bar{n})^{2}}=C \frac{I^{\prime}(x)}{B^{2}(x)} f(T), I^{\prime}(x)=I(x) x^{-3 / 2}, B^{\prime}(x)=\frac{B(x) x^{-3 / 2}}{[A(x)+B(x)]}
$$

where $C$ is a constant, $I(x)$ and $B(x)$ are the intensities at a height $x$ above the solar limb in the plane of the sky and intersecting the line of sight and $f(T)$ is a slowly varying function of $T$.

In Table 1, we have summarized the results of the analysis for two north polar regions. We find a good correlation between $\mathrm{B}(\mathrm{x})$ and $\mathrm{I}(\mathrm{x})$ (Figure 1) while Figure 2 presents the theoretical fit to observed profile of $B(x)$ for region $I$.

We find the irregularity to be greater than 1 and increasing with height for the two regions analyzed. In addition, the scale height temperature estimated from XUV observations is higher than the correspond-

E. R. Priest and V. Krishan (eds.), Basic Plasma Processes on the Sun, 253-254.

(C) 1990 IAU. Printed in the Netherlands. 
ing white-light scale height temperature. An increase in $X$ with height can account for this behavior. We have specifically chosen two regions that seemed relatively free of structures and we still obtained $X>1$. Thus the coronal irregularity at least in this region seems to be a feature of the inner corona. The detailed analysis of these data are in progress and results will be published elsewhere.

TABLE I. X inferred from observations of the North polar region

\begin{tabular}{lllccc}
\hline $\begin{array}{c}\text { Position } \\
\text { Angle }\end{array}$ & $\mathrm{n}=1.1$ & $\mathrm{x}=1.2$ & $\frac{\mathrm{x}(1.2)}{\mathrm{x}(1.1)}$ & $\begin{array}{c}\mathrm{T}_{\text {white }} \\
\text { in } 10^{6} \mathrm{~K}\end{array}$ & $\begin{array}{c}\mathrm{T}_{\mathrm{X}} \mathrm{VV} \\
\text { in } 10^{6} \mathrm{~K}\end{array}$ \\
\hline I. $356-0-5$ & $3.5 \pm 3.7$ & $4.3 \pm 4.5$ & 1.23 & 1.23 & 1.42 \\
II. $337-346$ & $1.32 \pm 1.7$ & $2.35 \pm 1.4$ & 1.78 & 1.08 & 1.40 \\
\hline
\end{tabular}
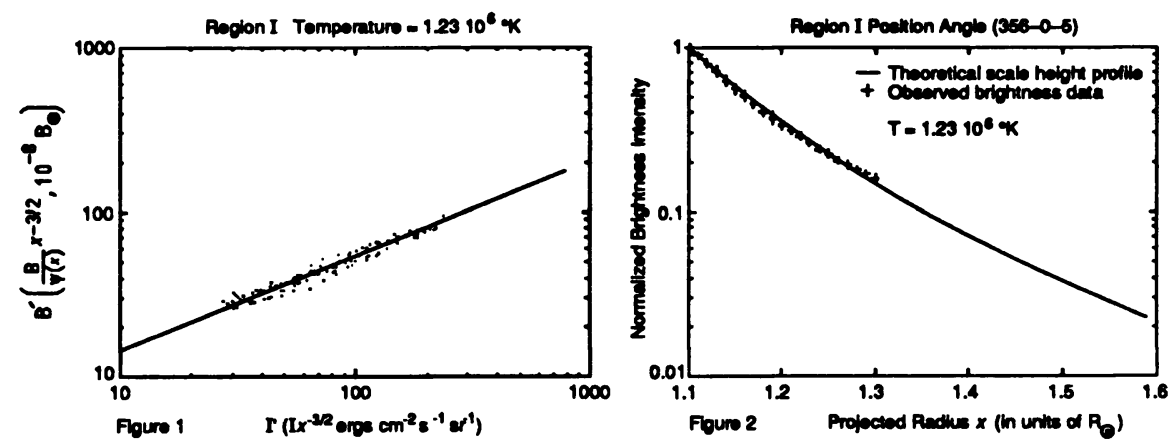

\section{References}

Fisher, R.R. (1989). 'In preparation'.

Guhathakurta, M. (1989). The large and small scale density structure of the solar corona. Ph.D. Thesis, University of Denver, Denver, Colorado.

Orrall, F.Q., Rottman, G.J., Fisher, R.R., and Munro, R.H. (1990). The solar coronal density irregularity $\left(\mathrm{n}^{2}\right) /(\mathrm{n})^{2}$ derived from simultaneous measurements of the EUV and $k$-coronal brightness. Ap.J., 349, in press.

Rottman, G.J. (1989). 'In preparation'. 